\title{
What we can learn from observations of cooling neutron stars
}

\author{
D. G. Yakovlev ${ }^{1}$ and P. Haensel ${ }^{2}$ \\ ${ }^{1}$ Ioffe Physical Technical Institute, Politekhnicheskaya 26, 194021 St.-Petersburg, Russia \\ 2 Copernicus Astronomical Center, Bartycka 18, 00-716 Warsaw, Poland \\ e-mail: haensel@camk.edu.pl
}

Received 3 September 2002 / Accepted 17 April 2003

\begin{abstract}
A generic toy model of a cooling neutron star (NS) is used to analyze cooling of NSs with nucleon and exotic compositions of the cores. The model contains the parameters which specify the levels of slow and fast neutrino emission as well as the lower and upper densities of the layer where the slow emission transforms into the fast one. The prospects to constrain these parameters from the present and future observations of isolated middle-aged NSs are discussed.
\end{abstract}

Key words. stars: neutron - dense matter

\section{Introduction}

Neutron stars (NSs) are compact stellar objects which contain matter of supranuclear density in their cores. The equation of state (EOS) of this matter cannot be calculated unambiguously (e.g., Lattimer \& Prakash 2001). Instead, there are many theoretical models which give a wide scatter of EOSs, from soft to stiff ones, with standard nucleon/hyperon or exotic compositions of matter. The nucleon matter consists mainly of neutrons (n) with an admixture of protons and electrons (p and e) and possibly muons. Some model EOSs predict also the appearance of hyperons. The exotic matter may contain pion condensates, kaon condensates, or quarks (or a mixture of these components). The indicated models are still almost not constrained by the observations of NSs.

In this paper we discuss the ability to constrain the EOS in the NS cores by confronting the observations of thermal emission from isolated NSs with the theory of NS cooling. In the last three decades the theory has been compared with the observations by many authors (e.g., Page 1998a) but the problem is complicated and the main features are still unknown (too many factors are involved, such as neutrino emission mechanisms, superfluidity of baryon components of matter; see, e.g., Yakovlev et al. 2002). Thus, at this stage it is sufficient to be general rather than accurate and use a simple toy model of cooling NSs. This will allow us to formulate the current status of the problem (Sects. 4 and 5) without complicated simulations.

\section{A toy model of a cooling NS}

Let us formulate our toy model.

A middle-aged NS $\left(t \lesssim 10^{5}\right.$ yr) cools mainly via neutrino emission from its core (the region of densities

Send offprint requests to: D. G. Yakovlev,

e-mail: yak@astro.ioffe.ru $\rho \gtrsim 1.5 \times 10^{14} \mathrm{~g} \mathrm{~cm}^{-3}$ ). We assume that the core can be subdivided into three zones: the outer zone, $\rho<\rho_{\mathrm{s}}$; the transition zone, $\rho_{\mathrm{s}} \leq \rho<\rho_{\mathrm{f}}$; and the inner zone, $\rho \geq \rho_{\mathrm{f}}$. If the central stellar density $\rho_{\mathrm{c}} \leq \rho_{\mathrm{s}}$, the two last zones are absent.

In the outer zone we assume slow neutrino emission while in the inner zone we assume fast emission with the neutrino emissivity $Q_{v}\left(\mathrm{erg} \mathrm{s}^{-1} \mathrm{~cm}^{-3}\right)$ :

$Q_{v}^{\text {slow }}\left(\rho \leq \rho_{\mathrm{s}}\right)=Q_{\mathrm{s}} T_{9}^{8}, \quad Q_{v}^{\text {fast }}\left(\rho \geq \rho_{\mathrm{f}}\right)=Q_{\mathrm{f}} T_{9}^{6}$.

Here, $T_{9}$ is the internal stellar temperature $T$ expressed in $10^{9} \mathrm{~K}$, while $Q_{\mathrm{s}}$ and $Q_{\mathrm{f}}$ are constants. For simplicity, we use a linear interpolation in $\rho$ between $Q_{v}^{\text {slow }}$ and $Q_{v}^{\text {fast }}$ in the transition zone. This approximation, although oversimplified, seems to be sufficient for exploring the main features of the transition zone - its position and thickness (which are presently unknown).

The proposed generic description of $Q_{v}$ covers a number of physical model EOSs of nucleon and exotic supranuclear matter with different leading neutrino processes collected in Tables 1 and 2. In these tables, $\mathrm{N}$ is a nucleon (n or $\mathrm{p}$ ); $v$ and $\bar{v}$ are neutrino and antineutrino; $q$ is a quasinucleon (mixed $n$ and $\mathrm{p}$ states); $\mathrm{u}$ and $\mathrm{d}$ are quarks.

For instance, $Q_{\mathrm{s}}$ can describe the modified Urca (Murca) process in nonsuperfluid nucleon matter, or weaker NNbremsstrahlung (e.g., nn-bremsstrahlung if Murca is suppressed by a strong proton superfluidity as considered by Kaminker et al. 2001, 2002). The factor $Q_{\mathrm{f}}$ can describe the processes of fast neutrino emission: a powerful direct Urca (Durca) process in nucleon matter (Lattimer et al. 1991) or weaker (but nevertheless strong) Durca-like processes in exotic phases of matter (pion condensed, kaon condensed, or quark matter) as reviewed, e.g., by Pethick (1992). The neutrino emission from hyperon matter is qualitatively the same as from nucleon matter. The bottom line of Table 2 refers to nonsuperfluid quark matter in NS cores. 
Table 1. Main processes of slow neutrino emission in nucleon matter: Murca and bremsstrahlung (brems).

\begin{tabular}{|c|c|c|}
\hline Process & & $Q_{\mathrm{s}}, \operatorname{erg~\mathrm {cm}^{-3}\mathrm {s}^{-1}}$ \\
\hline Murca & $\mathrm{nN} \rightarrow \mathrm{pNe} \bar{v} \quad \mathrm{pNe} \rightarrow \mathrm{nN} \nu$ & $10^{20}-3 \times 10^{21}$ \\
\hline Brems. & $\mathrm{NN} \rightarrow \mathrm{NN} v \bar{v}$ & $10^{19}-10^{20}$ \\
\hline
\end{tabular}

The transition zone mimics an onset of the fast neutrino emission with growing $\rho$. In nonsuperfluid matter, the lower density $\rho_{\mathrm{s}}$ is a threshold density of the fast emission; the threshold is usually sharp, i.e., $\rho_{\mathrm{f}} \approx \rho_{\mathrm{s}}$. In realistic models of superfluid matter, the fast emission turns on gradually, and the transition zone may be broader (e.g., Yakovlev et al. 2002). The broadening is caused by superfluidity provided its strength is high at the formal (nonsuperfluid) fast emission threshold and decreases at higher $\rho$. Then the superfluidity strongly suppresses the fast emission at the formal threshold and starts to open it at some higher $\rho=\rho_{\mathrm{s}}$, where the superfluid suppression ceases to be very strong. It fully opens the fast emission at still higher $\rho=\rho_{\mathrm{f}}$, where the superfluid suppression is almost completely removed.

To be specific, we adopt a density profile within the NS in the form: $\rho(r)=\rho_{\mathrm{c}}\left[1-(r / R)^{2}\right]$, where $R$ is the NS radius. According to Lattimer \& Prakash (2001), this is a reasonable approximation for many realistic NS models. Then the NS (gravitational) mass is $M=8 \pi \rho_{\mathrm{c}} R^{3} / 15$.

To follow the NS cooling we solve the equation of thermal balance in the approximation of isothermal interior (e.g., Glen \& Sutherland 1980):

$C\left(T_{i}\right) \frac{\mathrm{d} T_{\mathrm{i}}}{\mathrm{d} t}=-L_{v}^{\infty}\left(T_{\mathrm{i}}\right)-L_{\gamma}^{\infty}\left(T_{\mathrm{s}}\right)$,

where $T_{\mathrm{s}}$ is the effective surface temperature, $T_{\mathrm{i}}(t)=T(r, t) \mathrm{e}^{\Phi}$ is the redshifted internal temperature which is constant throughout the isothermal interior $\left(\rho \gtrsim \rho_{\mathrm{b}} \sim 10^{10} \mathrm{~g} \mathrm{~cm}^{-3}\right)$ with account for the effects of General Relativity; $T(r, t)$ is the local internal temperature of matter, and $\Phi(r)$ is the metric function (describing gravitational redshift). Furthermore, $C$ is the total NS heat capacity, $L_{\gamma}^{\infty}=4 \pi \sigma T_{\mathrm{s}}^{4} R^{2}\left(1-r_{\mathrm{g}} / R\right)$ is the photon surface luminosity as detected by a distant observer $\left(r_{\mathrm{g}}=2 G M / c^{2}\right.$ being the gravitational radius), and $L_{\gamma}^{\infty}$ is the redshifted neutrino luminosity. We have

$C\left(T_{i}\right)=\int \mathrm{d} V c(T), \quad L_{v}^{\infty}\left(T_{i}\right)=\int \mathrm{d} V Q_{v}(T) \mathrm{e}^{2 \Phi}$,

where $c(T)$ is the heat capacity per unit volume, $\mathrm{d} V$ is an element of proper volume; integration is done over the NS interior. In addition, we introduce $T_{\mathrm{s}}^{\infty}=T_{\mathrm{s}} \sqrt{1-r_{\mathrm{g}} / R}$, the effective surface temperature detected by a distant observer.

Taking the simplicity of the toy model, we assume $\mathrm{d} V=$ $4 \pi r^{2} \mathrm{~d} r$ (flat space) and $\mathrm{e}^{\Phi}=\sqrt{1-r_{\mathrm{g}} / R}$ (constant redshift) in the NS interior. With the above assumptions on $Q_{v}(T)$, the function $L_{v}^{\infty}(T)$ is calculated in an analytic form.

We employ $c(T)=c_{0}(T) f_{\mathrm{C}}$, where $c_{0}(T)$ is the heat capacity of degenerate baryon matter composed of one particle species of number density $n=\rho / m_{\mathrm{N}}\left(m_{\mathrm{N}}\right.$ being the bare nucleon mass) and effective mass $m_{\mathrm{N}}^{*}=0.7 m_{\mathrm{N}} ; f_{\mathrm{C}}$ is the parameter introduced to absorb the drawbacks of our toy model and to
Table 2. Leading processes of fast neutrino emission in nucleon matter and three models of exotic matter.

\begin{tabular}{llll}
\hline \hline Model & Process & & $Q_{\mathrm{f}}, \mathrm{erg} \mathrm{cm}^{-3} \mathrm{~s}^{-1}$ \\
\hline Nucleon matter & $\mathrm{n} \rightarrow \mathrm{pe} \bar{v}$ & $\mathrm{pe} \rightarrow \mathrm{n} v$ & $10^{26}-10^{27}$ \\
Pion condensate & $\mathrm{q} \rightarrow \mathrm{qe} \bar{v}$ & $\mathrm{qe} \rightarrow \mathrm{q} v$ & $10^{23}-10^{26}$ \\
Kaon condensate & $\mathrm{q} \rightarrow \mathrm{qe} \bar{v}$ & $\mathrm{qe} \rightarrow \mathrm{q} v$ & $10^{23}-10^{24}$ \\
Quark matter & $\mathrm{d} \rightarrow \mathrm{ue} \bar{v}$ & $\mathrm{ue} \rightarrow \mathrm{d} v$ & $10^{23}-10^{24}$ \\
\hline
\end{tabular}

account for the effects of other particles and superfluidity. The total NS heat capacity is then also evaluated in an analytic form: $C=4 \pi \times 0.2293 R^{3} c_{\mathrm{c}}(T)$, where $c_{\mathrm{c}}(T)$ is the specific heat at the stellar center. In the NS models with $Q_{\mathrm{s}}>10^{20}$ (appropriate for nonsuperfluid nucleons, see above) we set $f_{\mathrm{C}}=1.25$ adding thus $25 \%$ contribution of the heat capacity of protons (Page 1994). In the models with $Q_{\mathrm{s}}<10^{20}$ (appropriate to strongly superfluid protons at $\rho_{\mathrm{c}}<\rho_{\mathrm{s}}$ ) we set $f_{\mathrm{C}}=1.0$ at $\rho_{\mathrm{c}}<\rho_{\mathrm{s}}$ and $f_{\mathrm{C}}=1.25$ at $\rho_{\mathrm{c}}>\rho_{\mathrm{s}}$. In fact, the value of $f_{\mathrm{C}}$ weakly affects the cooling curves at the neutrino cooling stage and such variations of $f_{\mathrm{C}}$ might be neglected: they do not change our principal results.

We adopt the formula of Potekhin et al. (1997) to relate the internal and the surface temperatures (for the standard NS heat blanketing envelopes made of iron). The surface gravity in this expression has been calculated as $g=G M \mathrm{e}^{-\Phi} / R^{2}$, i.e., including the General Relativity effects.

Thus we obtain a toy model of cooling NSs in a closed form. The model contains five parameters: $Q_{\mathrm{s}}, Q_{\mathrm{f}}, \rho_{\mathrm{s}}, \rho_{\mathrm{f}}$, and $f_{\mathrm{C}}$. Similar models have been used, e.g., by Lattimer et al. (1994) and Page (1998a, 1998b) who have studied the sensitivity of the NS cooling to the efficiency of fast neutrino emission (i.e., to variations of $Q_{\mathrm{f}}$ ). They implemented the generic model of $Q_{v}^{\text {fast }}(T)$ into exact cooling codes. Our toy model is much simpler but it enables us to take a general view of the problem (by varying additionally $Q_{\mathrm{s}}, \rho_{\mathrm{s}}$, and $\rho_{\mathrm{f}}$ ) and mimic thus a number of physical phenomena without complicated computation.

\section{Cooling models}

For an adopted EOS of dense matter (for a set of five parameters, in our case) we can construct a sequence of NS models with different $\rho_{\mathrm{c}}$ (i.e., different $M$ ). We can mimic the stiffness of EOSs by choosing different mass-radius relations. For simplicity, we take $R=12 \mathrm{~km}$ for all models (the approximation of constant $R$ may hold in a wide range of $M$ for a number of EOSs, see Lattimer \& Prakash 2001). The main results will be the same for more realistic mass-radius relations. We will vary $\rho_{\mathrm{c}}$ from $7 \times 10^{14} \mathrm{~g} \mathrm{~cm}^{-3}$ to $1.4 \times 10^{15} \mathrm{~g} \mathrm{~cm}^{-3}$ thus varying $M$ from $1.02 M_{\odot}$ to $2.04 M_{\odot}$.

The calculations predict three types of cooling NSs: (1) low-mass NSs, $M<M_{\mathrm{s}}\left(M_{\mathrm{s}}\right.$ corresponds to the central density $\left.\rho_{\mathrm{c}}=\rho_{\mathrm{s}}\right)$; (2) medium-mass NSs, $M_{\mathrm{s}}<M \lessgtr M_{\mathrm{f}}\left(M_{\mathrm{f}}\right.$ corresponds to $\rho_{\mathrm{c}} \sim \rho_{\mathrm{f}}$ ); and (3) high-mass NSs, $M \gtrsim M_{\mathrm{f}}$. The same conclusions have been made by Kaminker et al. (2002) with regard to the models of NSs with nucleon cores.

Low-mass NSs are slowly cooling objects since their neutrino emission is slow: $Q_{v}=Q_{v}^{\text {slow }}$. Their cooling curves are 


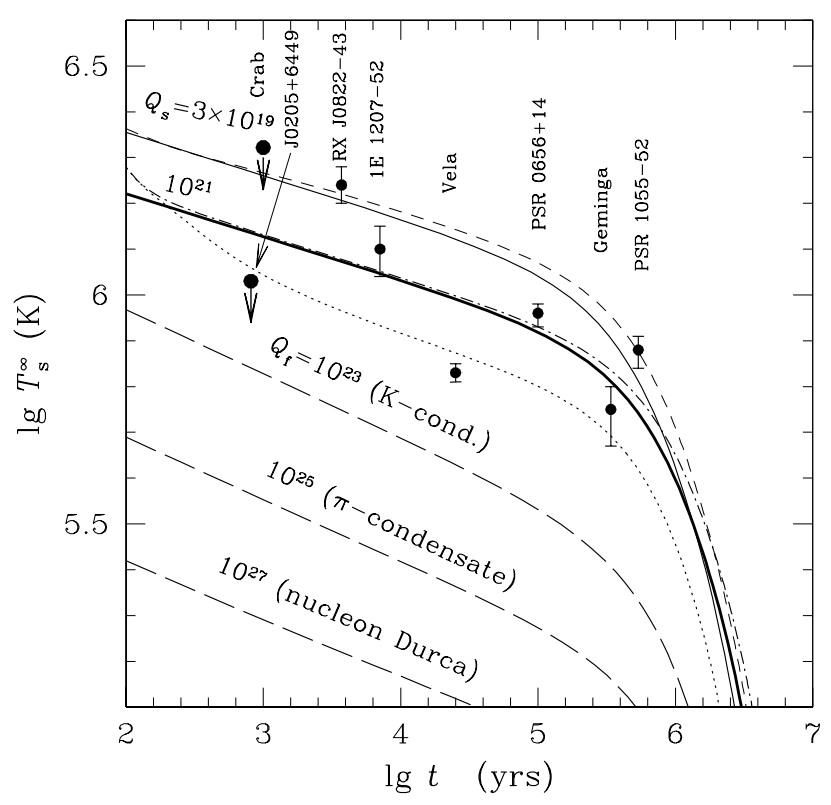

Fig. 1. Observational limits on surface temperatures of eight NSs versus toy-model cooling curves of low-mass NSs (solid lines) with two slow neutrino emission levels and high-mass NSs (long dashes) with three fast neutrino emission levels. Dashed-and-dot line: slow cooling of a nonsuperfluid 1.4 $M_{\odot}$ NS calculated with the exact cooling code; dotted line: the same but assuming triplet-state neutron pairing in the core, Takatsuka (1972) model; short-dashes: the same but with strong proton pairing and nonsuperfluid neutrons.

almost insensitive to $M$ for obvious reasons: both $L_{v}$ and $C$ increase with increasing $M$ but this increase is compensated in the ratio $L_{v} / C$ which determines the cooling rate at the neutrino-cooling stage $\left(L_{v} \gg L_{\gamma}\right)$.

High-mass NSs cool mainly via fast neutrino emission, $Q_{v}=Q_{v}^{\text {fast }}$, from the inner zones. Their cooling curves are also not too sensitive to $M$. These rapidly cooling middle-aged stars are noticeably colder than the slowly cooling ones.

Medium-mass NSs show cooling intermediate between the slow and fast ones. While increasing $M$ from $M_{\mathrm{s}}$ to $M_{\mathrm{f}}$ and higher, we get a sequence of cooling curves which realize the transition from the slow to the fast cooling regimes.

The thick and thin solid lines in Fig. 1 display typical cooling curves of low-mass NSs $\left(\rho_{\mathrm{c}}=8 \times 10^{14} \mathrm{~g} \mathrm{~cm}^{-3}, M=\right.$ $1.16 M_{\odot}$ ) with two levels of the slow neutrino emission, $Q_{\mathrm{s}}$. The long-dashed lines show cooling curves for high-mass NSs $\left(\rho_{\mathrm{c}}=1.4 \times 10^{15} \mathrm{~g} \mathrm{~cm}^{-3}, M=2.04 M_{\odot}, \rho_{\mathrm{s}}=8 \times 10^{14} \mathrm{~g} \mathrm{~cm}^{-3}\right.$, $\rho_{\mathrm{f}}=10^{15} \mathrm{~g} \mathrm{~cm}^{-3}$ ) with three levels of the fast neutrino emission, $Q_{\mathrm{f}}=10^{23}, 10^{25}$ and $10^{27}$, which roughly correspond to NSs with kaon condensates, pion condensates, and nucleon matter with Durca process in NS cores. NSs with hyperon cores are expected to cool at about the same rate as NSs with nucleon cores. One can see the great difference of cooling scenarios at various $Q_{\mathrm{s}}$ and $Q_{\mathrm{f}}$.

The thick solid curve $\left(Q_{\mathrm{s}}=10^{21}\right)$ is very close to those obtained (e.g., Page 1998a, 1998b; Kaminker et al. 2002) for low-mass non-superfluid NSs with the nucleon cores using exact cooling codes. It may be regarded as the basic slow-cooling curve.

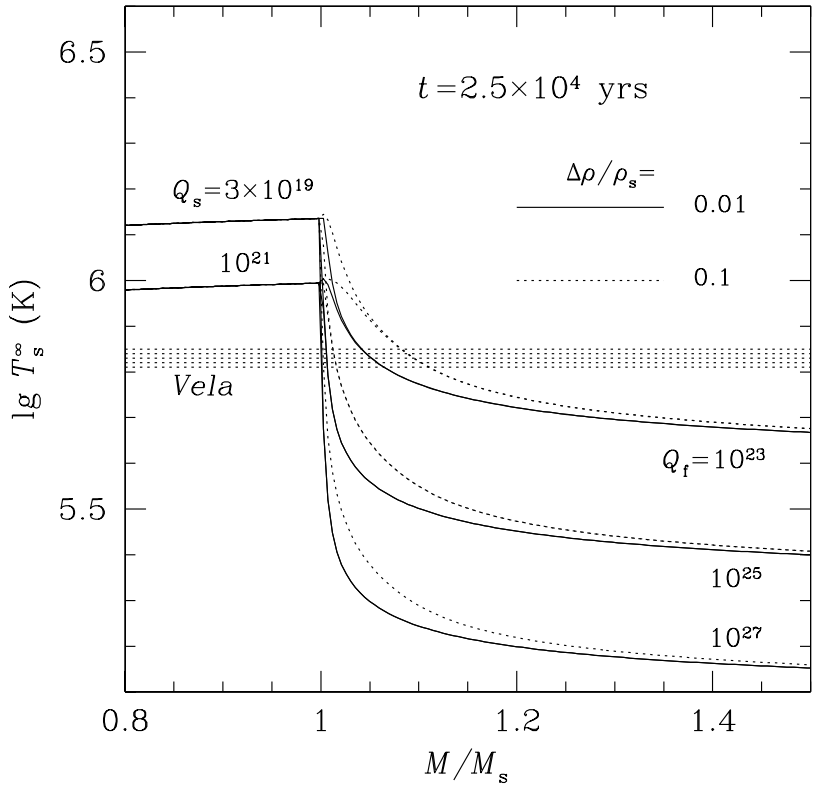

Fig. 2. Transition from slow to fast cooling with increasing NS mass over the threshold value $M_{\mathrm{s}}$ for the same values of $Q_{\mathrm{s}}$ and $Q_{\mathrm{f}}$ as in Fig. 1 and two transition density intervals, $\Delta \rho / \rho_{\mathrm{s}}=0.01$ or 0.1 , at a fixed NS age $t=25000$ yrs. Shaded strip: observational limits for the Vela pulsar.

For comparison, we present three cooling curves calculated with an exact cooling code for a 1.4 $M_{\odot}$ NS (the nucleon core with forbidden Durca process, $\rho_{\mathrm{c}}=1.22 \times 10^{15} \mathrm{~g} \mathrm{~cm}^{-3}, R=$ $11.65 \mathrm{~km}$, EOS B in the notation of Kaminker et al. 2002). The dot-and-dashed curve refers to a nonsuperfluid star and agrees with the basic slow-cooling curve. The short-dashed curve is for a NS with strong proton superfluidity (which switches off the proton heat capacity and the neutrino reactions involving protons); it agrees with the upper solid curve. By varying the heat capacity parameter $f_{\mathrm{C}}$ of our toy model, we could get even better agreement with the two exact cooling curves indicated above. At $t \lesssim 100 \mathrm{yr}$ the exact code accurately describes the NS thermal relaxation which is not reproduced by the toy model. At these $t$, the exact cooling curves noticeably deviate from the toy-model ones. The third exact curve is explained later.

Figure 2 shows the transition from slow to fast cooling with increasing $M$ for the same $Q_{\mathrm{s}}$ and $Q_{\mathrm{f}}, \rho_{\mathrm{s}}=8 \times 10^{14} \mathrm{~g} \mathrm{~cm}^{-3}$ $\left(M_{\mathrm{s}}=1.16 M_{\odot}\right)$, and two relative density widths of the transition zone $\Delta \rho / \rho_{\mathrm{s}} \equiv\left(\rho_{\mathrm{f}}-\rho_{\mathrm{s}}\right) / \rho_{\mathrm{s}}=0.01$ or 0.1 . The first value of $\Delta \rho / \rho_{\mathrm{s}}$ corresponds to a sharp threshold of the fast emission, while the second value is appropriate to a smooth threshold. The NS age is fixed, $t=25000$ yrs (the age of the Vela pulsar, Lyne et al. 1996). The main features of Fig. 2 would not change if we varied $\rho_{\mathrm{s}}$ from $8 \times 10^{14}$ to $1.2 \times 10^{15} \mathrm{~g} \mathrm{~cm}^{-3}\left(M_{\mathrm{s}}\right.$ from $1.16 M_{\odot}$ to $\left.1.75 M_{\odot}\right)$. If the contrast $Q_{\mathrm{f}} / Q_{\mathrm{s}}$ between the fast and slow emissivities is not too high $\left(Q_{\mathrm{f}} / Q_{\mathrm{s}} \lessgtr 10^{3}\right)$, the transition is rather smooth even for a very narrow density width $\Delta \rho$. For higher contrasts, the transition is smooth only if the density width is not too small, $\Delta \rho / \rho_{\mathrm{s}} \gtrsim 0.1$. 


\section{Confronting theory with observations}

Although the toy model is oversimplified, it reproduces the main features of exact calculations and can be confronted with the observations of thermal emission from cooling NSs.

The observational basis is shown in Fig. 1. It displays the observational values of $T_{\mathrm{s}}^{\infty}$ for six middle-aged isolated NSs, the same as in Yakovlev et al. (2002) excluding RX J1856-3754 and RX J0002+62. The latter two sources are most interesting but the interpretation of the observed spectra and the extraction of the surface thermal radiation is complicated (as discussed, e.g., in Pavlov et al. 2002; Walter \& Lattimer 2002, and in references therein with regard to RX J1856-3754; as commented by Pavlov 2002 with regard to RX J0002+62). The objects presented in Fig. 1 are: RX J0822-43, 1E 1207-52, Vela, PSR 0656+14, Geminga, and PSR 1055-52. The data on $T_{\mathrm{s}}^{\infty}$ and $t$ for these NSs are taken from the sources cited in Kaminker et al. (2002). We display also the upper limit of $T_{\mathrm{s}}^{\infty}$ for the Crab pulsar (Tennant et al. 2001) and for PSR J0205+6449 in the supernova remnant 3C 58 (Slane et al. 2002).

The comparison of the data with the cooling curves leads to the following generic conclusions.

(1) The hottest observed NS, RX J0822-43, lies well above the basic slow-cooling curve but is compatible with the models of low-mass NSs with $Q_{\mathrm{s}} \sim 3 \times 10^{19}$, i.e., with the reduced slow neutrino emission. These conclusions have been made, e.g., by Kaminker et al. (2001, 2002). Alternatively, one can assume either additional reheating mechanisms in the stellar interiors (which will complicate the theory) or the presence of accreted NS envelopes (which increase the electron thermal conductivity and make the surface layers hotter at the neutrino cooling stage, see e.g. Potekhin et al. 1997; Page 1998a).

(2) The coldest observed NSs, particularly the Vela and Geminga pulsars, lie well below the basic slow-cooling curve. They require enhanced neutrino emission in their cores, with $Q_{\mathrm{f}} \gtrsim 10^{23}$, but we cannot pinpoint the nature of this enhancement. This conclusion has been made by several authors (e.g., Page 1998a, 1998b). Even a weak enhancement produced by kaon condensates would be consistent with the data, but any stronger enhancement produced by pion condensates or nucleon Durca is also allowed. Thus, future search for colder NSs would be crucial but it may take time because of difficulties in detecting faint sources.

(3) A rather uniform scatter of observational points between the hottest and coldest NSs indicates a sufficiently smooth transition from slow to fast cooling, i.e., the existence of a representative class of medium-mass NSs. This circumstance has been mentioned, e.g., by Kaminker et al. (2001, 2002) with regard to the cooling of NSs with nucleon cores.

(4) The threshold density of fast neutrino emission, $\rho_{\mathrm{s}}$, can be placed anywhere in the interval from $\sim 8 \times 10^{14} \mathrm{~g} \mathrm{~cm}^{-3}$ to $\sim 1.2 \times 10^{15} \mathrm{~g} \mathrm{~cm}^{-3}$. For any $\rho_{\mathrm{s}}$ from this interval we can build a sequence of models of medium-mass NSs. Tuning their masses, we could explain the data. Adopting various $\rho_{\mathrm{s}}$ and $\rho_{\mathrm{f}}$ we will attribute different masses to the same sources, as seen from Fig. 2 with the Vela pulsar as an example. Similar conclusions were made by Kaminker et al. (2001, 2002) for cooling NSs with nucleon cores. Their transition layer was produced by the weakening of the proton pairing with increasing $\rho$ and the associated broadening of the Durca threshold.

(5) For a not too high contrast of slow and fast neutrino emissivities $\left(Q_{\mathrm{f}} / Q_{\mathrm{s}} \lesssim 10^{3}\right)$ we can build a representative class of medium-mass NSs even with a narrow density width of the transition zone, $\Delta \rho / \rho_{\mathrm{s}} \ll 1$. For sharper contrasts, the density width has to be sufficiently wide, $\Delta \rho / \rho_{\mathrm{s}} \gtrsim 0.1$, as follows from the cooling simulations of superfluid NSs with nucleon cores (Kaminker et al. 2001, 2002). One needs a wide transition zone to obtain a representative class of medium-mass NSs with superfluid nucleon or pion-condensed cores. This is possible in the presence of a strong superfluidity in the vicinity of the fastemission threshold.

\section{Discussion and conclusions}

Thus, our generic cooling analysis (5-parameter physics input leading to the families of cooling curves of NSs with different $M$ ) is too flexible to fix the nature of the fast neutrino emission and its density threshold. Consequently, the data can be explained by a number of physical EOSs of dense matter. Notice that the cooling curves are more sensitive to the composition of matter than to the stiffness of the EOS (although the composition and stiffness are actually interrelated).

For instance, Kaminker et al. (2001, 2002) exploit the idea of nucleon matter in the NS core with the onset of the Durca process at high densities. They assume the presence of a strong proton superfluidity at not too high densities to suppress the Murca process and broaden the Durca threshold. The suppression of Murca allows them to reduce the slow neutrino emission level $Q_{\mathrm{s}}$ (from about $10^{21}$ to about $3 \times 10^{19}$ ) and explain thus the hottest observed sources (Fig. 1). The broadening of the Durca threshold ensures a representative class of medium-mass NSs to interpret cooler objects.

In the phenomenological approach of Kaminker et al. $(2001,2002)$ the crucial ${ }^{1} \mathrm{~S}_{0}$ proton superfluidity is modeled by a specific density dependence of the proton critical temperature $T_{\mathrm{cp}}$. Consider, for instance, a particular realization of this model applied by Yakovlev et al. (2002) to analyze the thermal state of PSR J0205+6449. For this specific model (model 1p with EOS A, in notations of the authors), the maximum of $T_{\text {cp }}\left(\sim 7 \times 10^{9} \mathrm{~K}\right)$ is reached at $5.75 \times 10^{14} \mathrm{~g} \mathrm{~cm}^{-3}$. The critical temperature $T_{\mathrm{cp}}$ decreases at higher densities, but it is still $\approx 4.1 \times 10^{9} \mathrm{~K}$ at the Durca threshold, $\rho_{\mathrm{D}}=7.85 \times 10^{14} \mathrm{~g} \mathrm{~cm}^{-3}$, and then drops to zero at about $1.2 \rho_{\mathrm{D}}$. Can $T_{\mathrm{cp}}$ be as high as $\sim 4 \times 10^{9} \mathrm{~K}$ at $\rho=\rho_{\mathrm{D}}$ where the proton fraction is about $11 \%$ ? In a recent paper Tsuruta et al. (2002) argue that this would contradict the existing microscopic models of nucleon superfluidity, because the proton number density $n_{\mathrm{p}}$ is too large at $\rho=\rho_{\mathrm{D}}$ to allow for the ${ }^{1} \mathrm{~S}_{0}$ proton pairing. Taking the model of Yakovlev et al. (2002) mentioned above we get $n_{\mathrm{p}}=$ $0.047 \mathrm{fm}^{-3} \approx 0.3 n_{0}$ at $\rho=\rho_{\mathrm{D}}$, i.e., noticeably smaller than $n_{0}=0.16 \mathrm{fm}^{-3}$, the nucleon number density in saturated nuclear matter. For the same number density of neutrons in neutron matter, the critical temperature of the ${ }^{1} \mathrm{~S}_{0}$ neutron superfluidity is $\sim 1.3 \times 10^{10} \mathrm{~K}$ if a realistic bare $\mathrm{NN}$ interaction is used 
(Lombardo \& Schulze 2001). The medium effects (polarization effects, self-energy corrections) can decrease it to $7 \times 10^{9} \mathrm{~K}$ or lower, depending on a particular model. In the case of the proton component of the neutron-star matter the np interaction can decrease the proton effective mass, lowering further $T_{\mathrm{cp}}$. However, this does not mean that the value $T_{\mathrm{cp}}\left(\rho_{\mathrm{D}}\right) \gtrsim 7 \times 10^{9} \mathrm{~K}$, followed by a rapid drop of $T_{\mathrm{cp}}$ with increasing $\rho$, is ruled out by contemporary microscopic theories. In contrast to the case of neutron matter at $n_{\mathrm{n}}<n_{0}$, reliable calculation of $T_{\mathrm{cp}}\left(\rho_{\mathrm{D}}\right)$ in the neutron-star matter, starting from a realistic NN interaction and including the medium effects, remains still a challenge for the many-body theory. Therefore, we think that the use of a "minimal model" (npe matter with Durca acting at $\rho>\rho_{\mathrm{D}}$, combined with the appropriate nucleon superfluidity) remains a valid first-step approach.

Notice that our general assumption on the neutrino emissivity $Q_{v}(T, \rho)$ would be violated in the presence of $\left({ }^{3} \mathrm{P}_{2}\right)$ neutron superfluidity in the NS core with a density dependent critical temperature $T_{\mathrm{cn}}(\rho)$ which has the maximum in the range from $\sim 10^{8}$ to $\sim 2 \times 10^{9} \mathrm{~K}$ (see, e.g., Kaminker et al. 2002). The neutrino emission due to Cooper pairing of neutrons will then be so strong that it will initiate a really fast cooling even at $M<M_{\mathrm{s}}$, violating the interpretation of relatively hot and old sources, first of all PSR 1055-52. As an example, by the dotted line in Fig. 1 we show the cooling of $1.4 M_{\odot}$ NS with the nucleon core and forbidden Durca process in the presence of neutron superfluidity (model of Takatsuka 1972, with maximum $T_{\mathrm{c}}$ of about $9 \times 10^{8} \mathrm{~K}$ at $\rho \approx 5 \times 10^{14} \mathrm{~g} \mathrm{~cm}^{-3}$ ). One can see that NSs with this superfluidity would be too cold to explain the data.

Another physical model of cooling NSs was presented by Tsuruta et al. (2002). It is based on pion condensation at supranuclear densities exploiting a similar idea: slow cooling of low-mass NSs and faster cooling of massive NSs. Their cooling curve of low-mass NS $\left(1.2 M_{\odot}\right.$, nucleon core, forbidden Durca process) agrees with our basic slow-cooling curve (after translating our curve, $T_{\mathrm{s}}^{\infty}(t)$, to their format, $\left.L_{\gamma}^{\infty}(t)\right)$. Note that Tsuruta et al. (2002) misplaced the positions of some observational data in their figure. Most important is RX J0822-43. They (as well as we) take the data from Zavlin et al. (1999) who give $L_{\gamma}^{\infty}=(7.1-10.1) \times 10^{33} \mathrm{erg} \mathrm{s}^{-1}\left(\lg L_{\gamma}^{\infty}=33.85-34.00\right)$ while Tsuruta et al. present $\lg L_{\gamma}^{\infty} \approx 33.57-33.97$. Thus, RX J0822-43 is sufficiently warm and cannot be explained with the basic slow-cooling model (Fig. 1). Moreover, according to Tsuruta et al., they employ the model neutron superfluidity of Takatsuka (1972). Therefore, their curve (if properly calculated) should resemble our dotted curve, and their model would then disagree with a number of observational limits. To avoid this disagreement one can change the model of nucleon superfluidity. A natural model of rather strong proton superfluidity and weak neutron superfluidity at $\rho \sim(3-8) \times 10^{14} \mathrm{~g} \mathrm{~cm}^{-3}$ considered by Kaminker et al. $(2001,2002)$ seems to be the most suitable.

Acknowledgements. The authors are grateful to an anonymous referee, A. D. Kaminker, and K. P. Levenfish for useful critical remarks. This work was supported in part by the RFBR (grants 02-02-17668 and 03-07-90200) and KBN (grant 5 P03D 020 20).

\section{References}

Glen, G., \& Sutherland, P. 1980, ApJ, 239, 671

Kaminker, A. D., Haensel, P., \& Yakovlev, D. G. 2001, A\&A, 373, L17

Kaminker, A. D., Yakovlev, D. G., \& Gnedin, O. Y. 2002, A\&A, 383, 1076

Lattimer, J. M., \& Prakash, M. 2001, ApJ, 550, 426

Lattimer, J. M., Pethick, C. J., Prakash, M., \& Haensel, P. 1991, Phys. Rev. Lett., 66, 2701

Lattimer, J. M., van Riper, K. A., Prakash, M., \& Prakash, M. 1994, ApJ, 425, 802

Lombardo, U., \& Schulze, H.-J. 2001, in Physics of Neutron Star Interiors, ed. D. Blaschke, N. K. Glendenning, \& A. Sedrakian (Lecture Notes in Physics, Berlin: Springer), 30

Lyne, A. G., Pritchard, R. S., Graham-Smith, F., \& Camilo, F. 1996, Nature, 381, 497

Page, D. 1994, ApJ, 428, 250

Page, D. 1998a, in The Many Faces of Neutron Stars, ed. R. Buccheri, J. van Paradijs, \& M. A. Alpar (Dordrecht: Kluwer), 539

Page, D. 1998b, in Neutron Stars and Pulsars, ed. N. Shibazaki, N. Kawai, S. Shibata, \& T. Kifune (Tokyo: Universal Academy Press), 183

Pavlov, G. G. 2002, private communication

Pavlov, G. G., Zavlin, V. E., \& Sanwal, D. 2002, in Proc. of 270 Heraeus Seminar on Neutron Stars, Pulsars and Supernova Remnants, ed. W. Becker, H. Lesh, \& J. Trümper (Garching: MPE), 273

Pethick, C. J. 1992, Rev. Mod. Phys., 64, 1133

Potekhin, A. Y., Chabrier, G., \& Yakovlev, D. G. 1997, A\&A, 323, 415

Slane, P., Helfand, D. J., \& Murray, S. S. 2002, ApJ, 571, L45

Takatsuka, T. 1972, Prog. Theor. Phys., 48, 1517

Tennant, A. F., Becker, W., Juda, M., et al. 2001, ApJ, 544, L173

Tsuruta, S., Teter, M. A., Takatsuka, T., Tatsumi, T., \& Tamagaki, R. 2002, ApJ, 571, L143

Walter, F. M., \& Lattimer, J. 2002, ApJ, 576, L145

Yakovlev, D. G., Kaminker, A. D., Gnedin, O. Y., \& Haensel, P. 2001, Phys. Rep., 354, 1

Yakovlev, D. G., Gnedin, O. Y., Kaminker, A. D., \& Potekhin, A. Y. 2002, in Proc. of 270 Heraeus Seminar on Neutron Stars, Pulsars and Supernova Remnants, ed. W. Becker, H. Lesh, \& J. Trümper (Garching: MPE), 287

Yakovlev, D. G., Kaminker, A. D., Haensel, P., \& Gnedin, O. Y. 2002, A\&A, 389, L24

Zavlin, V. E., Trümper, J., \& Pavlov, G. G. 1999, ApJ, 525, 959 